\title{
CORRELACIÓN MORFO-ESPECÍFICA EN FLORES DE PALICOUREA PADIFOlIA (RUBIACEAE)
}

\author{
Angélica Hernández y Juan Francisco Ornelas ${ }^{1}$ \\ Departamento de Ecología y Comportamiento Animal, Instituto de Ecología, A.C., Km 2.5 Carretera Antigua a \\ Coatepec No. 351, Congregación El Haya, Apdo. Postal 63, Xalapa, Ver. C.P. 91070. Tel (52) (228) 842-1800 \\ ext. 4121, Fax (52) (228) 818-7809 \\ ${ }^{1}$ Autor para la correspondencia. Correo-e: ornelasj@ecologia.edu.mx
}

\begin{abstract}
Resumen: La distilia es un polimorfismo floral en donde aproximadamente la mitad de las plantas en una población tienen los estilos largos y los estambres cortos (Pin) y la otra mitad estilos cortos y estambres largos (Thrum). Se ha sugerido que la posición relativa de anteras y estigmas en las flores distílicas promueve el flujo de polen entre flores de morfos opuestos (polinización legítima), en comparación con el flujo de polen entre flores del mismo morfo (polinización ilegítima). Estudiamos la morfología floral de ambos morfos (seis variables) en Palicourea padifolia (Rubiaceae) y encontramos diferencias significativas entre morfos florales en los rasgos que definen al polimorfismo floral. Sin embargo, las relaciones entre los rasgos morfológicos de las flores difieren entre morfos florales (morfo-específico) y las relaciones significativas fueron más fuertes en el morfo con los estilos cortos. Los patrones de correlación entre algunos de los rasgos florales sugieren presiones de selección diferencial entre morfos florales, independientemente del tamaño de la corola.
\end{abstract}

Palabras clave: colibríes, distilia, Palicourea, polimorfismo floral.

\begin{abstract}
Distyly is a floral polymorphism in which about half of the plants in a population have long styles and short stamens (Pin), whereas the remainder possess short styles and long stamens (Thrum). It has been suggested that the relative position of anthers and stigmas in distylous flowers enhances pollen flow between flowers of opposite morphs (legitimate pollination), in comparison to pollen flow between flowers of the same morph (illegitimate pollination). We studied the floral morphology of both floral morphs (six variables) in Palicourea padifolia (Rubiaceae) and found significant differences between floral morphs in floral traits that define the floral polymorphism. However, the correlations between morphological traits of flowers differed between floral morphs (morph-specific), and the significant correlations were stronger in the shortstyled morph. The morph-specific correlation patterns among some of the floral traits suggest differential selective pressures between floral morphs, regardless of corolla length.
\end{abstract}

Keywords: distyly, floral polymorphism, hummingbirds, Palicourea.

$\mathbf{L}$ a extraordinaria diversidad de flores de las angiospermas es el producto del cambio evolutivo a partir de una base reducida de diseños florales de donde se han originado cambios morfológicos repetidos. La forma, el tamaño y el número de flores son aspectos importantes de diversificación morfológica entre las plantas con flores (Worley y Barrett, 2000; Galen y Cuba, 2001). A lo largo de la evolución de las angiospermas, es posible que las estructuras florales que originalmente aparecieron para una función determinada hayan cambiado completamente su función original, lo que dificulta la identificación de factores que moldearon su evolución (Walker-Larsen y Harder, 2000). Por ello, es importante explorar los factores que puedan explicar la variación en la morfología floral de muchas especies de angiospermas para lograr un entendimiento integral de la evolución floral. La mayoría de los estudios se han enfocado en el entendimiento de la fuerza de selección de los polinizadores sobre la morfología floral y la heredabilidad de los rasgos florales (Galen, 1989; Johnston, 1991; Campbell, 1996; Caruso, 2000). Sin embargo, pocos estudios han considerado otros factores como explicaciones alternativas a la variación en los rasgos florales (Galen y Cuba, 2001). Esto se debe a que muchos de los rasgos florales están intercorrelacionados (Cheverud, 1984; Clark, 1987), y de esa manera pueden aumentar o disminuir la adecuación de la planta (Roff, 1996).

Las correlaciones de los rasgos florales pueden mostrar un patrón específico ya que las flores se desarrollan como un órgano funcional integrado. Sin embargo, el patrón de correlación entre los rasgos puede estar relacionado intrínsecamente con el desarrollo y, por lo tanto, puede restringir la fuente de variación en estos patrones (hipótesis 
de restricción de desarrollo). Alternativamente, la selección impuesta por los polinizadores puede alterar el patrón de correlación fenotípica de los rasgos florales (hipótesis selectiva), los cuales pueden ser importantes en la adecuación de las plantas y adaptación a su ambiente (Worley y Barrett, 2000).

Los patrones de correlación en flores tubulares polinizadas por animales dependerán de la posición relativa del estigma y de las anteras con respecto a la apertura de la flor (Conner y Sterling, 1995; Conner, 1997). Conner y Via (1993) han sugerido que los polinizadores pueden promover una selección que incremente la correlación entre los rasgos florales de este tipo de flores, logrando una mayor correspondencia morfológica con el cuerpo de los polinizadores, y afectar directamente la eficiencia de la polinización (Thomson y Stratton, 1985; Harder y Barrett, 1993; Motten y Stone, 2000; Nishihiro et al., 2000). La dificultad para probar estas hipótesis controlando los posibles efectos filogenéticos (Herrera, 1992) es atribuible al inconveniente de encontrar grupos de plantas cercanamente emparentadas sujetas al mismo patrón de desarrollo floral por ancestría común, pero que además estén sujetos al mismo régimen de restricción de selección (polinizadores).

Las plantas distílicas pueden ser un modelo idóneo para probar estas hipótesis, ya que sus morfos florales están aparentemente sujetos al mismo patrón de desarrollo y comparten polinizadores. La distilia es un polimorfismo floral en donde aproximadamente la mitad de las plantas en una población tienen estilos largos y estambres cortos (Pin) y la otra mitad estilos cortos y estambres largos (Thrum) (Barrett, 1992). Darwin (1877) propuso que la posición recíproca de anteras y estigmas en flores distílicas promueve el flujo de polen entre flores de morfos opuestos, proceso que está mediado por los polinizadores (Beach y Bawa, 1980). Con la finalidad de explorar las hipótesis anteriormente planteadas, estudiamos la morfología floral de ambos morfos en Palicourea padifolia (Rubiaceae) y sus patrones de correlación. Si los patrones de correlación de los rasgos difieren entre morfos florales (morfo-específico), entonces pensaríamos que ambos morfos podrían estar expuestos a presiones de selección diferencial, ya que esas diferencias en sus correlaciones puede promover un flujo de polen asimétrico mediado por los polinizadores y esto, a la larga, promovería diferencias en su éxito reproductivo. Por el contrario, si los patrones de correlación de rasgos florales no difieren entre morfos florales, entonces pensaríamos en restricciones de desarrollo y que la morfología floral de $P$. padifolia estaría bajo las mismas presiones de selección y con un flujo simétrico de polen entre morfos.

\section{Materiales y métodos}

Sitio de estudio. Trabajamos en un área adyacente al Parque Ecológico Francisco Xavier Clavijero (30 ha) a $2.5 \mathrm{~km}$ de

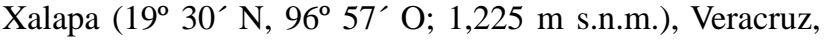
México. El parque es uno de los pocos remanentes de bosque mesófilo de montaña en la región central de Veracruz. La precipitación media anual es de $1,492.4 \mathrm{~mm}$, con una precipitación mínima en diciembre $(44.8 \mathrm{~mm})$ y un máximo en junio $(273.4 \mathrm{~mm})$. La temperatura media anual es de $18^{\circ} \mathrm{C}$, con una máxima en mayo $\left(20.4^{\circ} \mathrm{C}\right)$ y una mínima en enero $\left(14.9^{\circ} \mathrm{C}\right)$.

Sistema. Palicourea padifolia (Roem. et Schult.) C.M. Taylor et Lorence (Rubiaceae) se distribuye desde el sur de México hasta Panamá (Taylor, 1989). Es un arbusto (2 a 7 m de altura) común del sotobosque, autoincompatible, con inflorescencias piramidales y flores tubulares de color amarillo que abren un solo día (Taylor, 1989; Ree, 1997; Contreras y Ornelas, 1999). Hemos estimado que hay más de 500 individuos reproductivos en el área del Parque Clavijero (Ornelas et al., en prensa a). Cada individuo produce alrededor de 40 inflorescencias y en cada inflorescencia típicamente abren tres flores de manera asincrónica por día durante el periodo de floración que va de marzo a agosto (Ornelas et al., en prensa a). Cada flor tiene sólo dos óvulos. Nuestra población es morfológicamente distílica con igual proporción de morfos y presenta un sistema de incompatibilidad, en donde sólo flores polinizadas con polen proveniente del morfo opuesto producen semillas viables (Contreras y Ornelas, 1999). El tamaño de la corola, de las anteras y del lóbulo del estigma es mayor en las flores del morfo Thrum. En contraste, las flores del morfo Pin producen dos veces más granos de polen de menor tamaño que las del morfo Thrum (Ree, 1997; Contreras y Ornelas, 1999; Ornelas et al., en prensa a). Las flores del morfo Pin producen significativamente más néctar (media $\pm \mathrm{EE}, 13.99 \pm 0.77$ $\mu \mathrm{l} /$ flor) que las del morfo Thrum (10.24 $\pm 0.85 \mu \mathrm{l} /$ flor $)$ (Ornelas et al., en prensa b). Las flores son visitadas por 11 especies de colibríes (Campylopterus curvipennis, $C$. hemileucurus, Colibri thalassinus, Anthracothorax prevostii, Amazilia candida, A. cyanocephala, A. beryllina, A. yucatanensis, Lampornis amethystinus, Eugenes fulgens y Atthis heloisa), mariposas, abejas solitarias y abejorros (Ornelas et al., en prensa a). Las flores son consumidas por las aves Saltator atriceps y Chlorospingus ophthalmicus e infestadas por larvas de dípteros (Contreras y Ornelas, 1999). Los colibríes A. cyanocephala son los visitantes más frecuentes (30\% de las visitas por planta) y los polinizadores más eficientes de las flores de P. padifolia (Ornelas et al., en prensa a). Los frutos inician su desarrollo en julio y de manera asincrónica van madurando hasta diciembre (C. González, J.F. Ornelas y L. Jiménez, datos no publicados). Los frutos contienen típicamente dos semillas. El morfo Thrum produce casi el doble de frutos por infrutescencia con respecto al morfo Pin, independientemente del tamaño del pico del polinizador (Ornelas et al., en prensa a). 


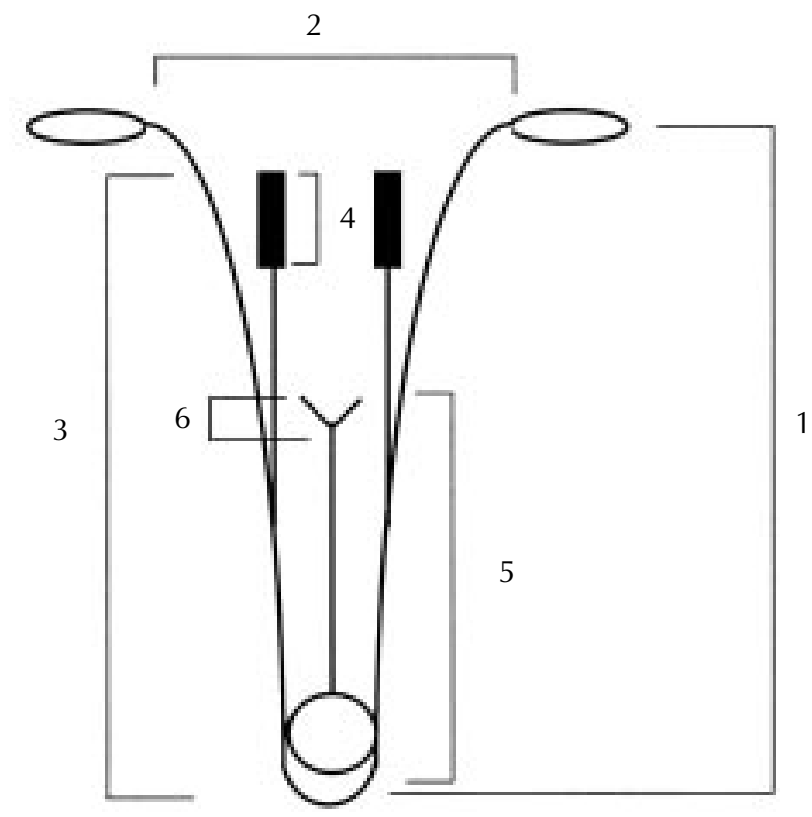

Figura 1. Diagrama que ilustra los rasgos florales medidos en Palicourea padifolia (Rubiaceae). (1) largo de corola, (2) ancho de la corola (apertura), (3) altura de la antera, (4) longitud de la antera, (5) altura del estigma, (6) longitud del lóbulo del estigma.

Muestreo. El presente estudio se realizó en junio de 2000, durante el pico de floración de la especie. Cuatro flores por planta del morfo Pin (30 plantas) y cuatro del morfo Thrum (30 plantas) fueron recolectadas a lo largo de la vereda principal del parque. En cada flor medimos seis rasgos: (1) longitud de la corola, (2) ancho de la corola, (3) altura de la antera, (4) longitud de la antera, (5) altura del estigma y (6) longitud del lóbulo del estigma (figura 1) con un vernier digital. Sólo una de las anteras por flor fue medida. Por último, calculamos la reciprocidad hercogámica como la diferencia de las alturas de los órganos sexuales entre morfos florales (altura del estigma - altura de la antera).

Análisis estadístico. La variación en los rasgos morfológicos entre morfos florales fue analizada con análisis de varianza (ANDEVA) para cada uno de los rasgos medidos empezando por un MANDEVA ya que las variables medidas pueden estar intercorrelacionadas. Usando un MANDEVA seguido de ANDEVAs de una vía reduce la probabilidad de inflar la tasa de error de Tipo I. En el modelo, el morfo floral fue considerado como un factor fijo y la variación entre individuos se anidó en el morfo floral. Para definir patrones de correlación de rasgos florales, promediamos los valores de los rasgos en cada flor seleccionada por planta y luego los transformamos al logaritmo base 10 para realizar un análisis de regresión simple entre todos los pares de rasgos florales por morfo floral. Una vez realizado esto, distinguimos entre las variables morfológicas que presentaron una correlación significativa en uno o dos de los morfos florales y comparamos las pendientes de las rectas entre morfos florales con una prueba de $t$ (Zar, 1984). Para los casos en los que detectamos una correlación significativa con la longitud de la corola, controlamos este posible efecto alométrico calculando los residuos de las relaciones y volvimos a hacer los análisis de regresión simple por morfo floral, ajustando los valores de significancia $(P)$ por comparaciones múltiples con una prueba de Bonferroni.

\section{Resultados}

Rasgos florales. El efecto del morfo sobre la variación en la morfología floral fue estadísticamente significativa (Wilks' Lambda $\left.=0.07, F_{7,226}=458.1, P=0.0001\right)$ pero no la variación entre individuos (Wilks' Lambda $=0.83, F_{42,1063.5}$ $=1.05, P=0.39$ ). Por lo tanto, los resultados de ANDEVAs seguidos del MANDEVA indican que la población estudiada

Cuadro 1. Media (mm), error estándar (EE), valores de $F$ y de significancia $(P)$ de ANDEVAs para la comparación de los rasgos florales entre morfos Pin y Thrum en Palicourea padifolia (Rubiaceae).

\begin{tabular}{|c|c|c|c|c|c|c|}
\hline \multirow[t]{2}{*}{ Rasgo floral } & \multicolumn{2}{|c|}{ Pin } & \multicolumn{2}{|c|}{ Thrum } & \multicolumn{2}{|c|}{ ANDEVA } \\
\hline & Media & $\mathrm{EE}$ & Media & $\mathrm{EE}$ & $F_{1,232}$ & $P$ \\
\hline Longitud corola & 14.69 & 0.10 & 16.49 & 0.18 & 61.93 & 0.0001 \\
\hline Apertura corola & 3.16 & 0.06 & 3.65 & 0.05 & 30.82 & 0.0001 \\
\hline Altura antera & 12.10 & 0.08 & 14.31 & 0.12 & 237.81 & 0.0001 \\
\hline Longitud antera & 3.74 & 0.03 & 3.48 & 0.04 & 28.82 & 0.0001 \\
\hline Altura estigma & 17.10 & 0.17 & 6.86 & 0.12 & 979.68 & 0.0001 \\
\hline Longitud lóbulo del estigma & 0.66 & 0.02 & 1.94 & 0.03 & 1596.49 & 0.0001 \\
\hline Hercogamia recíproca & 3.08 & 0.17 & 5.23 & 0.13 & 108.39 & 0.0001 \\
\hline
\end{tabular}


de P. padifolia presenta diferencias significativas entre morfos en la altura de estigma y de las anteras de las flores (cuadro 1). La hercogamia recíproca fue diferente estadísticamente y de mayor magnitud en el morfo Thrum. Las corolas del morfo Thrum fueron significativamente más largas que las del morfo Pin. El lóbulo de los estigmas de las flores Thrum fue significativamente más largo que el de las flores Pin. Es importante notar que las anteras en Thrum se encuentran a la altura de la apertura del tubo floral, mientras que en Pin el estigma sobresale de la corola (figura 2). La altura de la antera de un morfo con la altura del estigma del morfo opuesto (hercogamia recíproca) fue estadísticamente diferente; no observamos una reciprocidad perfecta entre las alturas de los órganos sexuales (figura 2). La separación entre estigmas y anteras dentro de cada morfo (hercogamia) fue más pronunciada en Thrum que en Pin (figura 2; cuadro 1).

Correlaciones morfológicas. En general, los coeficientes de correlación de Pearson fueron positivos y más altos en Thrum en las relaciones de los rasgos florales explorados. De las 42 relaciones examinadas, una en Pin y siete en Thrum fueron significativamente diferentes de cero después de haber ajustado los valores de significancia por comparaciones múltiples (cuadro 2). Observamos una relación significativa entre la longitud de la corola y la altura de la antera en ambos morfos florales (figura 3a). Sin embargo, la relación fue más fuerte en Thrum $(r=0.8)$ que en Pin $(r=0.5)(t=-4.99$, $P<0.001)$. La correlación entre la longitud de la corola y la altura del estigma sólo se observó en Thrum $(t=43.09$, $P<0.001$; figura 3b). Además, detectamos una relación significativamente positiva entre la longitud de la corola y la longitud de la antera en Thrum (cuadro 2). También encontramos que la altura del estigma se correlacionó significativamente con la longitud de la antera en Thrum. Un punto importante de resaltar es la relación morfoespecífica entre los valores de hercogamia y la mayoría de los rasgos medidos en el morfo Thrum (cuadro 2).

\section{Discusión}

Rasgos florales. Las diferencias entre morfos florales observados con respecto al tamaño de las corolas (largo y ancho), lóbulos del estigma, y de las anteras corresponden a rasgos que se han considerado como accesorios al dimorfismo

Cuadro 2. Coeficiente de correlación de Pearson $(r)$ y probabilidades asociadas $(P)$ para los rasgos florales medidos en flores Pin (a) y flores Thrum (b) de Palicourea padifolia. Los asteriscos al lado de los valores de $P$ corresponden a un valor de significancia $\leq 0.001$ después de corregir por múltiples comparaciones usando una prueba de Bonferroni. (1) Rasgos relacionados positivamente con la longitud de la corola. LC = longitud corola, $\mathrm{AA}=$ altura antera, $\mathrm{AE}=$ altura estigma, $\mathrm{AC}=$ ancho corola, $\mathrm{LE}=$ longitud lóbulo estigma, $\mathrm{LA}=$ longitud antera, $\mathrm{HR}=$ hercogamia recíproca.

\begin{tabular}{|c|c|c|c|c|c|c|c|c|}
\hline & Rasgos florales & LC & AA & $\mathrm{AE}$ & $\mathrm{AC}$ & LE & $\mathrm{LA}$ & $\mathrm{HR}$ \\
\hline \multirow[t]{14}{*}{$\mathrm{a}$} & LC & & & & & & & \\
\hline & $\mathrm{AA}$ & 0.55 & & & & & & \\
\hline & & $0.001 *$ & & & & & & \\
\hline & $\mathrm{AE}$ & 0.06 & $0.18(1)$ & & & & & \\
\hline & & 0.75 & 0.33 & & & & & \\
\hline & $\mathrm{AC}$ & 0.45 & $0.14(1)$ & 0.11 & & & & \\
\hline & & 0.01 & 0.45 & 0.53 & & & & \\
\hline & LE & 0.05 & 0.11 & 0.07 & 0.23 & & & \\
\hline & & 0.76 & 0.54 & 0.71 & 0.20 & & & \\
\hline & $\mathrm{LA}$ & 0.17 & $0.02(1)$ & $0.38(1)$ & 0.11 & 0.16 & & \\
\hline & & 0.34 & 0.90 & 0.03 & 0.55 & 0.37 & & \\
\hline & $H R$ & 0.032 & 0.12 & 0.13 & 0.15 & 0.10 & 0.12 & \\
\hline & & 0.89 & 0.17 & 0.14 & 0.09 & 0.25 & 0.17 & \\
\hline & Rasgos florales & LC & $\mathrm{AA}$ & $\mathrm{AE}$ & $\mathrm{AC}$ & $\mathrm{LE}$ & $\mathrm{LA}$ & $\mathrm{HR}$ \\
\hline \multirow[t]{13}{*}{ b } & LC & & & & & & & \\
\hline & $\mathrm{AA}$ & 0.84 & & & & & & \\
\hline & & $<0.0001^{*}$ & & & & & & \\
\hline & $\mathrm{AE}$ & 0.72 & $0.10(1)$ & & & & & \\
\hline & & $<0.0001^{*}$ & 0.59 & & & & & \\
\hline & $\mathrm{AC}$ & 0.09 & $0.10(1)$ & 0.17 & & & & \\
\hline & & 0.62 & 0.59 & 0.36 & & & & \\
\hline & LE & 0.03 & 0.07 & 0.05 & 0.12 & & & \\
\hline & & 0.84 & 0.68 & 0.79 & 0.52 & & & \\
\hline & LA & 0.52 & $0.05(1)$ & $0.56(1)$ & 0.29 & 0.24 & & \\
\hline & & 0.0031* & 0.77 & $0.001 *$ & 0.10 & 0.18 & & \\
\hline & $H R$ & 0.62 & 0.44 & 0.81 & 0.07 & 0.02 & 0.44 & \\
\hline & & 0.0003* & $<0.0001^{*}$ & $<0.0001^{*}$ & 0.44 & 0.80 & $<0.0001 *$ & \\
\hline
\end{tabular}




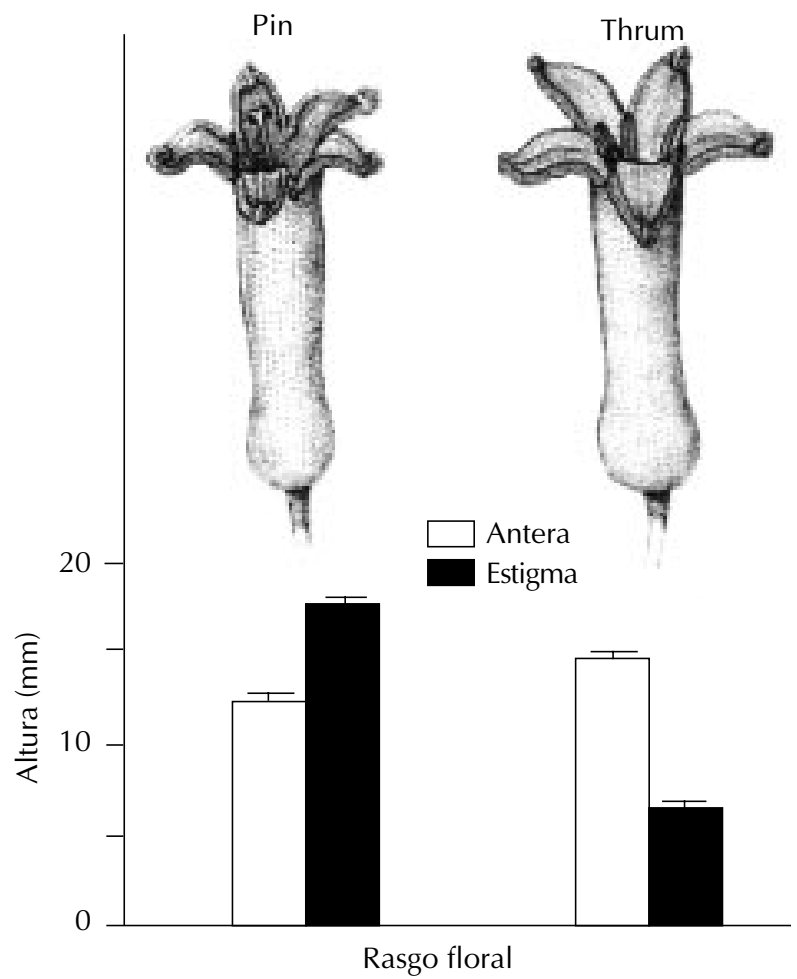

Figura 2. Separación antera-estigma (hercogamia) dentro y entre morfos florales (hercogamia recíproca) de Palicourea padifolia. Los valores indican medias y EE (ver cuadro 1 ).

floral. Al respecto, se ha planteado que éstos podrían contribuir al funcionamiento del sistema heterostílico (Ganders, 1979a; Dulberger, 1992). Sin embargo, muchas de las especies heteroestílicas pueden o no presentar este tipo de rasgos accesorios al dimorfismo floral y el significado funcional de los mismos aún no ha sido esclarecido (Barrett, 1992). Por ejemplo, nosotros encontramos que las anteras de las flores Pin son más largas que las Thrum en P. padifolia. Este dimorfismo también ha sido reportado en otras especies distílicas, pero también se ha observado el patrón inverso (Dulberger, 1992). Dado que el morfo Pin presenta un mayor número de granos de polen pero de menor tamaño con respecto a Thrum (Contreras y Ornelas, 1999), es posible que el mayor tamaño de las anteras en Pin permita contener un mayor número de granos de polen, semejante a lo observado en las especies tristílicas Pontederia y Lythrum (Barrett, 1992).

Con respecto al tamaño de la corola, se ha observado que en varias especies distílicas el morfo Thrum presenta corolas grandes (Pailler y Thompson, 1997; Ree, 1997; Contreras y Ornelas, 1999). Sin embargo, este rasgo no es generalizado ya que se ha observado que el morfo Pin puede presentar flores de mayor tamaño o ser similar el tamaño de las corolas en ambos morfos florales (Ganders, 1979b; Pailler y Thompson, 1997). Por otro lado, observamos que los lóbulos del estigma de las flores del morfo Thrum son más grandes que las de Pin. Este dimorfismo tampoco es generalizado ni presenta la misma dirección dentro de las especies distílicas reportadas en la literatura (Dulberger y Ornduff, 2000). La diferencia en el tamaño del estigma entre morfos es alta en $P$. padifolia, en comparación con otras especies distílicas (Dulberger, 1992; Dulberger y Ornduff, 2000; Ornelas et al., en prensa a), y la mayor área receptiva en Thrum puede promover un mayor flujo de polen legítimo hacia sus estigmas menos accesibles (Dulberger, 1992; Ornelas et al., en prensa a).

Correlaciones morfológicas. Las relaciones alométricas que detectamos entre los rasgos morfológicos de las flores pueden cumplirse en uno o en ambos morfos florales. Sin embargo, la intensidad de la relación (pendiente) es significativamente diferente de cero en sólo 8 de las 42 correlaciones realizadas (cuadro 2). Observamos una relación fuerte entre la longitud de la corola y la altura del estigma en Thrum (cuadro 2). Esta relación no puede ser explicada en términos de restricción en el desarrollo, ya que el estigma es un órgano que no está inserto en la corola. Las razones de porqué en Thrum aumenta el tamaño del estigma conforme aumenta el de la corola no son claras, pero es posible que sea para mantener una

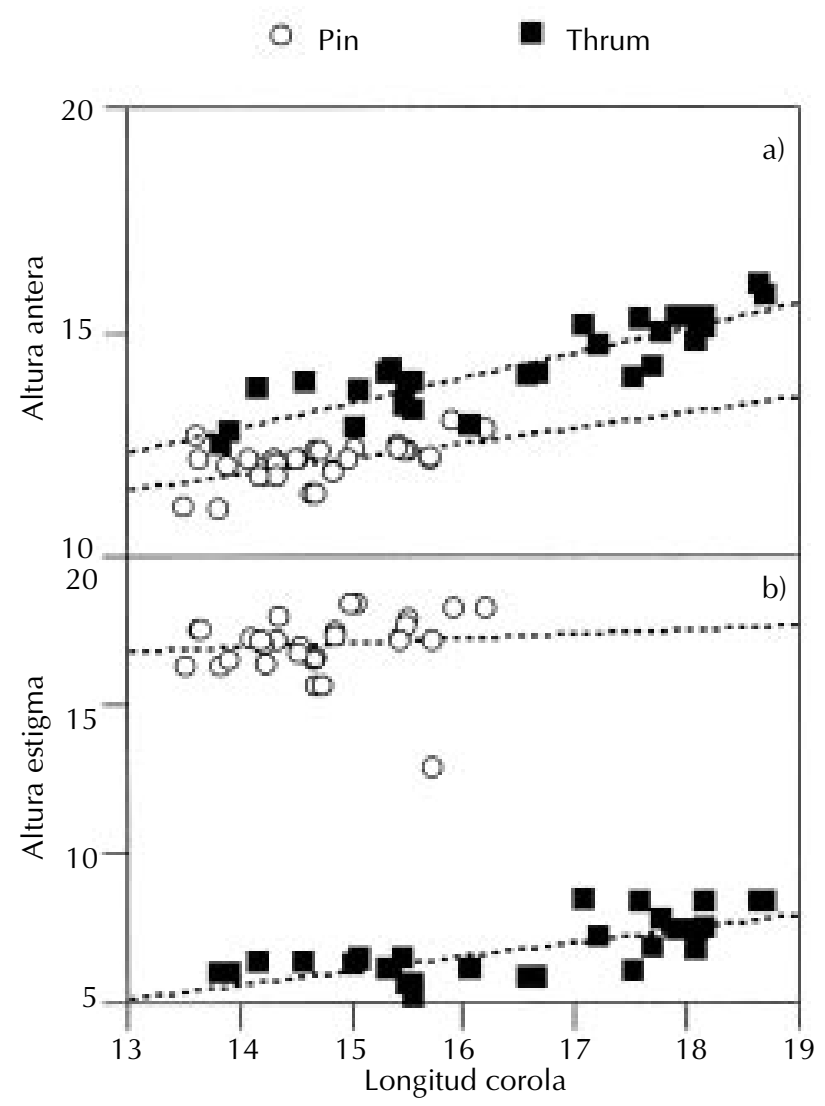

Figura 3. Relación de la longitud de la corola con (a) altura de la antera y (b) altura del estigma en milímetros por morfo floral en Palicourea padifolia. 
separación óptima entre el estigma y las anteras que aumente la captura de polen del morfo opuesto y evite la pérdida de su polen (Ganders, 1979b; Lloyd y Webb, 1992a,b; Barrett et al., 1996). También es posible que el patrón observado en Thrum pueda indirectamente resultar de presiones ejercidas por herbívoros que consumen el estigma exserto en Pin (Leege y Wolfe, 2002). Arroyo et al. (2002) plantean que el polimorfismo altura-estigma por sí solo posee un significado adaptativo, en donde el polimorfismo es el resultado de un balance ocasionado por diversas fuerzas selectivas que actúan sobre la transferencia de polen en ambas direcciones. Barrett et al. (2000) plantean que el dimorfismo altura-estigma no siempre está asociado a plantas heterostílicas y las fuerzas selectivas que lo mantienen son poco conocidas. En $P$. padifolia, es posible que exista una fuerza adicional y más intensa en Thrum que esté moldeando la altura del estigma de sus flores. La diferencia en el tamaño del lóbulo del estigma entre morfos florales en $P$. padifolia podría relacionarse indirectamente con diferencias en la probabilidad de recibir granos de polen provenientes del morfo opuesto. En apoyo a lo anterior, sabemos que en la población estudiada se presentan diferencias significativas en la carga de polen entre morfos florales (media $\pm \mathrm{EE}$; Pin $=14.4 \pm 3.1$ granos por estigma, Thrum $=26.5 \pm 3.4$ granos por estigma; $\mathrm{A}$. Hernández y J.F. Ornelas, datos no publicados), en dirección opuesta al patrón general en plantas distílicas (Dulberger, 1992), en donde los estigmas de Pin típicamente reciben cargas de polen mayores a los de Thrum. Esto sugiere que la relación observada entre la corola y la altura del estigma en Thrum puede promover una mayor deposición de polen en los estigmas más grandes de las flores Thrum (Ornelas et al., en prensa a).

La relación entre la longitud de la corola y la altura de la antera que detectamos en ambos morfos florales tiene también repercusiones importantes, ya que este tipo de relación puede modificar la reciprocidad entre los órganos florales de los morfos (cuadro 2). La relación significativa en ambos morfos puede deberse a que las anteras se encuentran insertadas en la corola de las flores. La relación es más fuerte en Thrum ya que este morfo presenta un mayor tamaño de la corola. Dulberger (1992) menciona que la inserción de las anteras en corolas tubulares puede favorecer una selección positiva sobre la longitud de la corola, lo cual promovería que las anteras se localizaran en una posición correcta con respecto al órgano funcional del morfo opuesto en plantas heterostílicas (Pailler y Thompson, 1997). Debido a esto, la elongación de las corolas en flores de un morfo puede modificar la reciprocidad de los órganos florales. De hecho, en nuestro estudio observamos que el morfo Thrum presentó un menor ajuste (reciprocidad) entre los órganos florales conforme aumenta el tamaño de la corola (cuadro 1 y 2). Por otro lado, hemos observado diferencias entre morfos florales en la proporción de granos de polen legítimos (del morfo opuesto) en los estigmas de las flores de $P$. padifolia (media \pm EE; Pin
$=0.2 \pm 0.03$ granos legítimos por estigma, Thrum $=0.5 \pm$ 0.05 granos legítimos por estigma; A. Hernández y J.F. Ornelas, datos no publicados), lo cual sugiere un mejor ajuste de la morfología floral de Thrum con respecto a sus polinizadores como lo sugiere los patrones de reciprocidad hercogámica en Thrum (cuadro 2). Por último, la relación positiva entre la altura del estigma y la longitud de la antera fue significativa sólo en Thrum (cuadro 2). Este resultado es consistente con la idea de que la relación entre la altura del estigma y la longitud de la antera esté favoreciendo un flujo de polen legítimo hacia Thrum (Barrett et al., 2000).

En conclusión, observamos un patrón de correlación morfoespecífico en la correlación de rasgos florales en $P$. padifolia. A pesar de que la variación en algunos de los rasgos depende del tamaño de las flores, los patrones de correlación de rasgos florales varían entre morfos. Observamos que las correlaciones son más fuertes en Thrum. Dado que la distilia está supuestamente determinada por una serie de genes agrupados por desequilibrio de ligamiento (supergene) (Barrett, 1992), la ausencia o menor intensidad en la correlación de rasgos florales en Pin podría sugerir que el supergene ha recombinado y que la selección está generando un cambio más rápido en este morfo. No sabemos si esto ha ocurrido en nuestra población, pero nuestros datos de deposición de polen apoyan la hipótesis de selección diferencial entre morfos. El papel de las diferencias en la intensidad de correlación de rasgos florales entre morfos sugiere que la evolución y funcionamiento de la distilia necesita mayor investigación.

\section{Agradecimientos}

Agradecemos a Victoria Sosa, Jorge A. Meave, y dos revisores anónimos por sus valiosos comentarios y sugerencias a versiones previas de este trabajo. Nuestro trabajo fue posible a través del apoyo de una beca doctoral del Consejo Nacional de Ciencia y Tecnología, México (No. 144021) a A. Hernández y del Departamento de Ecología y Comportamiento Animal, Instituto de Ecología, AC (Ref. 902-11-563). Este trabajo es parte de la tesis de doctorado de A. Hernández en el Programa de Ecología y Manejo de Recursos Naturales del posgrado del Instituto de Ecología, A.C.

\section{Literatura citada}

Arroyo J., Barrett S.C.H., Hidalgo R. y Cole W.W. 2002. Evolutionary maintenance of stigma-height dimorphism in Narcissus papyraceus (Amaryllidaceae). American Journal of Botany 89:1242-1249.

Barrett S.C.H. 1992. Evolution and function of heterostyly. Springer Verlag, Heidelberg.

Barrett S.C.H., Jesson L.K. y Baker A.M. 2000. The evolution and function of stylar polymorphisms in flowering plants. Annals of Botany 85:253-265. 
Barrett S.C.H., Lloyd D.G. y Arroyo J. 1996. Stylar polymorphism and evolution of heterostyly. En: Lloyd D.G y Barrett S.C.H. Eds. Floral Biology: Studies on Floral Evolution in Animal-pollinated Plants, pp. 339-376, Chapman and Hall, Nueva York.

Beach J.H. y Bawa K.S. 1980. Role of pollination in the evolution of dioecy from distyly. Evolution 34:1138-1142.

Campbell D.R. 1996. Evolution of floral traits in hermaphroditic plant: field measurements of heritabilities and genetic correlations. Evolution 50:1442-1453.

Caruso C.M. 2000. Competition for pollination influences selection on floral traits of Ipomopsis aggregata. Evolution 54:15461557.

Cheverud J.M. 1984. Quantitative genetics and developmental constraints on evolution by selection. Journal of Theoretical Biology 110:155-171.

Clark A.G. 1987. Genetic correlations: the quantitative genetics of evolutionary constraints. En: Loeschcke V. Ed. Genetic Constraints on Adaptative Evolution, pp. 25-45, Springer-Verlag, Berlín.

Contreras P.S. y Ornelas J.F. 1999. Reproductive conflicts of Palicourea padifolia (Rubiaceae) a distylous shrub of a tropical cloud forest in Mexico. Plant Systematics and Evolution 219:225-241.

Conner J.K. 1997. Floral evolution in wild radish: the roles of pollinators, natural selection, and genetic correlations among traits. International Journal of Plant Sciences 158:S108-S120.

Conner J.K. y Sterling A. 1995. Testing hypotheses of functional relationships: a comparative survey of correlation patterns among floral traits in five insect-pollinated plants. American Journal of Botany 82:1399-1406.

Conner J.K. y Via S. 1993. Patterns of phenotypic and genetic correlations among morphological and life-history traits in wild radish, Raphanus raphanistrum. Evolution 47:704-711.

Darwin C. 1877. The different forms of flowers on plants of the same species. Murray, Londres.

Dulberger R. 1992. Floral polymorphisms and their functional significance in the heterostylous syndrome. En: Barrett S.C.H. Ed. Evolution and Function of Heterostyly, pp. 41-77, Springer Verlag, Nueva York.

Dulberger R. y Ornduff R. 2000. Stigma morphology in distylous and non-heterostylous species of Villarsia (Menyanthaceae). Plant Systematics and Evolution 225:171-184.

Galen C.1989. Measuring pollinator-mediated selection on morphometric traits: bumblebees and the alpine sky pilot, Polemonium viscosum. Evolution 43:882-890.

Galen C. y Cuba J. 2001. Down the tube: pollinators, predators, and the evolution of flower shape in the alpine skipilot, $\mathrm{Po}$ lemonium viscosum. Evolution 55:1963-1971.

Ganders F.R. 1979a. The biology of heterostyly. New Zealand Journal of Botany 17:607-635.

Ganders F.R. 1979b. Heterostyly in Lithospermum cobrense (Boraginaceae). American Journal of Botany 66:746-748.

Harder L.D. y Barrett S.C.H. 1993. Pollen removal from tristylous Pontederia cordata: effects of anther position and pollinator specialization. Ecology 74:1059-1072.

Herrera C.M. 1992. Interspecific variation in fruit shape: allometry, phylogeny, and adaptation to dispersal agents. Ecology 73:18321841.

Johnston M.O. 1991. Natural selection on floral traits in two species of Lobelia with different pollinator. Evolution 45:1468-1479.

Leege L.M. y Wolfe L.M. 2002. Do floral herbivores respond to variation in flower characteristics in Gelsemium sempervirens (Loganiaceae), a distylous vine? American Journal of Botany 89:1270-1274.

Lloyd D. y Webb C. 1992a. The evolution of heterostyly. En: Barrett S.C.H. Ed. Evolution and Function of Heterostyly, pp. 151-178, Springer Verlag, Heidelberg.

Lloyd D. y Webb C. 1992b. The selection of heterostyly. En: Barrett S.C.H. Ed. Evolution and Function of Heterostyly, pp 179-207, Springer Verlag, Heidelberg.

Motten A.F. y Stone J.D. 2000. Heritability of stigma position and the effect of stigma-anther separation on outcrossing in a predominantly self-fertilizing weed, Datura stramonium (Solanaceae). American Journal of Botany 87:339-347.

Nishihiro J., Washitani I., Thomson J.D. y Thomson B.A. 2000. Patterns and consequences of stigma height variation in natural population of a distylous plant, Primula sieboldii. Functional Ecology 14:502-512.

Ornelas J.F., Jiménez L., González C. y Hernández A. En prensa a. Reproductive ecology of distylous Palicourea padifolia (Rubiaceae) in a tropical montane cloud forest. I. Hummingbirds' effectiveness as pollen vectors. American Journal of Botany.

Ornelas J.F., González C., Jiménez L., Lara C. y Martínez A. En prensa b. Reproductive ecology of distylous Palicourea padifolia (Rubiaceae) in a tropical montane cloud forest. II. Attracting and rewarding mutualistic and antagonistic visitors. American Journal of Botany.

Pailler T. y Thompson J.D. 1997. Distyly and variation in heteromorphic incompatibility in Gaernera vaginata (Rubiaceae) endemic to La Reunion Island. American Journal of Botany 67:95-103.

Ree R.H. 1997. Pollen flow, fecundity, and the adaptative significance of heterostyly in Palicourea padifolia (Rubiaceae). Biotropica 29:298-308.

Roff D.A. 1996. The evolution of genetic correlations: an analysis of patterns. Evolution 50:1392-1403.

Taylor C.M. 1989. Revision of Palicourea (Rubiaceae) in Mexico and Central America. Systematic Botany Monographs 26:1-102.

Thomson J.D. y Stratton D.A. 1985. Floral morphology and crosspollination in Erythronium grandiflorum (Liliaceae). American Journal of Botany 72:433-437.

Walker-Larsen J. y Harder L.D. 2000. The evolution of staminodes in angiosperms: patterns of stamen reduction, loss, and functional re-invention. American Journal of Botany 87:1367-1384.

Worley A.C. y Barrett S.C.H. 2000. Evolution of floral display in Eichhornia paniculata (Pontederiaceae): direct and correlated responses to selection on flower size and number. Evolution 54:1533-1545.

Zar J.H. 1984. Biostatistical analysis. Prentice Hall, Englewood Cliffs.

Fecha de recepción: 29 de abril de 2003

Versión corregida: 10 de noviembre de 2003

Aceptado: 10 de noviembre de 2003 
